\title{
Energy-Conserving Truncations for Convection with Shear Flow
}

\author{
Jean-Luc Thiffeault* and Wendell Horton ${ }^{\dagger}$ \\ Institute for Fusion Studies and University of Texas, Austin, Texas, 78712-1060
}

(October 30, 2018)

\begin{abstract}
A method is presented for making finite Fourier mode truncations of the Rayleigh-Bénard convection system that preserve invariants of the full partial differential equations in the dissipationless limit. These truncations are shown to have no unbounded solutions and provide a description of the thermal flux that has the correct limiting behavior in a steady-state. A particular loworder truncation (containing 7 modes) is selected and compared with the 6 mode truncation of Howard and Krishnamurti, 1 which does not conserve the total energy in the dissipationless limit. A numerical example is presented to compare the two truncations and study the effect of shear flow on thermal transport.
\end{abstract}

PACS numbers: 47.11.+j, 47.20.Bp, 47.27.-i, 47.27.Te

*e-mail: jeanluc@hagar.ph.utexas.edu

†e-mail: horton@hagar.ph.utexas.edu 


\section{INTRODUCTION}

In a horizontal layer of fluid with fixed higher temperature on the bottom boundary and fixed lower temperature on the top boundary, cellular convective flow occurs for a certain range of Rayleigh number $R$ and Prandtl number $\sigma$. ubiquitous in nature, occuring in slightly modified form in the atmosphere, the ocean, the earth's mantle, and in the convection zone of the interior of stars. It was originally believed that flows in a finite container should scale as its vertical dimension. However, experiments have shown that thermal convection in a horizontal layer of fluid heated from below can show motions spanning the largest horizontal dimension of the container, 3 known as shear flows. Since the experimental setting precluded any externally imposed shear forces, it is concluded that these shear flows are driven by a Reynolds stress tensor with non-vanishing horizontal average. This behavior is also seen in numerical experiments,

In this paper we make truncations of the Rayleigh-Bénard system using the Galerkin (or spectral) method. 0 The chosen basis is the standard Fourier one, because of its great simplicity and the fact that it is especially well-suited to the stress-free boundary conditions. One of the major points to be addressed is how to make a truncation that retains the truncated invariants of the full Partial Differential Equations (PDE's) in the dissipationless limit, and whether or not this has any effect on simulations in the dissipative case. We will show that energy-conserving truncations have several advantages over their non-energyconserving counterpart.

Section II is devoted to a presentation of the equations governing the system and the preserved quantities of the equations of motion in the dissipationless limit are displayed. In Section III we use the Galerkin method to expand the stream function and temperature field into a complete set of modes, deriving a set of coupled Ordinary Differential Equations (ODE's). The condition under which a mode truncation preserves the invariants of the dissipationless PDE's is obtained. Section [V presents a low-order model for shear flow generation, which is an extension of a model presented in Ref. 1. The model is compared 
with its predecessor and the advantages are explicitly shown. Finally, Section $\square$ is a summary of the main arguments of the paper.

\section{MODEL EQUATIONS}

The two-dimensional Rayleigh-Bénard problem for an incompressible fluid is governed in the Boussinesq approximation by

$$
\begin{aligned}
\frac{\partial \nabla^{2} \psi}{\partial t}+\left[\psi, \nabla^{2} \psi\right] & =\frac{\partial T}{\partial x}+\nu \nabla^{4} \psi, \\
\frac{\partial T}{\partial t}+[\psi, T] & =\frac{\partial \psi}{\partial x}+\kappa \nabla^{2} T
\end{aligned}
$$

where $\psi$ is the stream function, $T$ is the deviation of the temperature from a linear conduction profile, $\nu$ is the kinematic viscosity, and $\kappa$ is the thermal conductivity. All the quantities are dimensionless, the Prandtl number $\sigma$ is $\nu / \kappa$, and the Rayleigh number $R$ is $1 / \nu \kappa$. The horizontal coordinate is $x$ and the vertical one is $y$, with $(x, y) \in[0,2 \pi L] \times[0, \pi]$. (The choice $y \in[0, \pi]$, as opposed to $y \in[0,1]$, leads to a Rayleigh number smaller by a factor of $\pi^{4}$.) The Poisson bracket used is defined as $[A, B]=\partial_{x} A \partial_{y} B-\partial_{y} A \partial_{x} B$, and the velocity

field is given in terms of the stream function by $\mathbf{v}=\hat{\mathbf{z}} \times \nabla \psi=\left(-\partial_{y} \psi, \partial_{x} \psi\right)$. We assume the fluid is periodic in $x$ and has stress-free boundary conditions at the top and bottom walls:

$$
\psi=\nabla^{2} \psi=\partial_{x} \psi=T=0, \quad \text { for } y=0 \text { or } \pi
$$

In the dissipationless limit, $\nu=\kappa=0$, Eqs. (11) admit an infinite number of conserved quantities (this is a general feature of noncanonical infinite-dimensional Hamiltonian systems - for a discussion of the invariants of an analogous set of equations, see Ref. 6). Here we shall concern ourselves with the total energy, $E$, given by

$$
E=\frac{1}{2}\left\langle(\nabla \psi)^{2}\right\rangle-\langle y T\rangle=K+U
$$

where $K$ is the kinetic energy, $U$ is the potential energy, and the angle brackets denote the integral over the fluid domain. In the dissipationless limit, the time derivative of $E$ is 


$$
\begin{aligned}
\frac{d}{d t} E & =-\left\langle\psi \frac{\partial \nabla^{2} \psi}{\partial t}\right\rangle-\left\langle y \frac{\partial T}{\partial t}\right\rangle \\
& =\left\langle\psi\left(\left[\psi, \nabla^{2} \psi\right]-\partial_{x} T\right)\right\rangle+\left\langle y\left([\psi, T]-\partial_{x} \psi\right)\right\rangle \\
& =-\left\langle\psi \partial_{x} T\right\rangle+\langle\psi[T, y]\rangle=0,
\end{aligned}
$$

showing that the total energy is conserved (we have set surface terms to zero in Eq. (4). The total internal (thermal) energy of the fluid, $\langle T\rangle$, is also conserved.

\section{MODE EXPANSION}

In this Section we look at truncations of system (1) and their properties. In the first part we derive the equations of motion for modes of the truncations (Section [II A). In Section [IIB we examine the behavior of the invariants mentioned in Section 11 after they are truncated and show (in a manner similar to Ref. (7) that they can be made to remain invariant by adding certain modes to the system. Such truncations will be called energy-conserving. Finally, in Section $\mathbb{1 1 0}$ we examine the important properties of these truncations, namely that the truncated system has no singular solutions and that the thermal flux is properly modeled.

\section{A. Derivation of the ODE's}

To turn the system of partial differential equations (11) into ordinary differential equations, we use the following normal mode expansions for the $\psi$ and $T$ fields:

$$
\begin{aligned}
& \psi(x, y, t)=\sum_{(m, n) \in A_{\psi}} \psi_{m n}(t) e^{i(m \alpha x+n y)}, \\
& T(x, y, t)=\sum_{(m, n) \in A_{T}} T_{m n}(t) e^{i(m \alpha x+n y)},
\end{aligned}
$$

where $\alpha \equiv 1 / L$ is the inverse aspect ratio. The summations are over some sets $A_{\psi}$ and $A_{T}$ of modes (i.e., $(m, n)$ pairs, where both $m$ and $n$ can be negative or zero). If both of these sets are infinite and contain all possible $(m, n)$ pairs, then the equalities hold in (5); otherwise, 
the expansion is a truncation. Expansion (5) is more general than those used in Refs. 8,0,9 in two ways: first, it allows for a variable phase in the rolls (by allowing the $\psi_{m n}$ 's to be complex) and second, the expansion admits a non-vanishing shear flow part (the $\psi_{0 n}$ modes).

The reality of the fields and the stress-free boundary conditions (22) lead to

$$
\begin{aligned}
& \psi_{m n}=\psi_{-m,-n}^{*}=-\psi_{m,-n}, \\
& T_{m n}=T_{-m,-n}^{*}=-T_{m,-n},
\end{aligned}
$$

so that if, say, $\psi_{11}$ is in $A_{\psi}$, then so are $\psi_{1,-1}, \psi_{-1,1}$, and $\psi_{-1,-1}$, and similarly for $A_{T}$. Note that $A_{\psi}$ and $A_{T}$ need not contain the same modes. For convenience we define $\rho_{m n} \equiv \alpha^{2} m^{2}+n^{2}$ to be the eigenvalues of the operator $-\nabla^{2}$. If we insert Eqs. (50) into the Boussinesq equations (11), we obtain the following set of coupled nonlinear ODE's:

$$
\begin{aligned}
\frac{d}{d t} \psi_{m n}= & -\nu \rho_{m n} \psi_{m n}-i \frac{\alpha m}{\rho_{m n}} T_{m n} \\
& +\sum_{\substack{m^{\prime}+m^{\prime \prime}=m \\
n^{\prime}+n^{\prime \prime}=n}} \alpha\left(m^{\prime} n^{\prime \prime}-m^{\prime \prime} n^{\prime}\right) \frac{\rho_{m^{\prime \prime} n^{\prime \prime}}}{\rho_{m n}} \psi_{m^{\prime} n^{\prime}} \psi_{m^{\prime \prime} n^{\prime \prime}}, \\
\frac{d}{d t} T_{m n}= & -\kappa \rho_{m n} T_{m n}+i \alpha m \psi_{m n} \\
& +\sum_{\substack{m^{\prime}+m^{\prime \prime}=m \\
n^{\prime}+n^{\prime \prime}=n}} \alpha\left(m^{\prime} n^{\prime \prime}-m^{\prime \prime} n^{\prime}\right) \psi_{m^{\prime} n^{\prime}} T_{m^{\prime \prime} n^{\prime \prime}} .
\end{aligned}
$$

\section{B. Preservation of the Invariants}

The kinetic and potential energies of Eq. (3) have the expansions

$$
\begin{aligned}
& K=\frac{1}{2} \sum_{m, n} \rho_{m n}\left|\psi_{m n}\right|^{2}, \\
& U=i \sum_{p \neq 0} \frac{(-1)^{p}}{p} T_{0 p} .
\end{aligned}
$$

We now ask whether the total energy $E$ is still conserved in the dissipationless limit for a truncated system. Taking the time derivative of Eqs. (8) and using Eqs. (7) with $\nu=\kappa=0$, we obtain after some manipulation: 


$$
\begin{aligned}
\frac{d}{d t} E & =\frac{d}{d t}(K+U) \\
& =i \alpha \sum_{m, n} m \psi_{m n} T_{m n}^{*}+i \alpha \sum_{\substack{m, n \\
p \neq 0}}(-1)^{p} m \psi_{m n} T_{m, n-p}^{*} \\
& =i \alpha \sum_{m, n, p}(-1)^{p} m \psi_{m n} T_{m, n-p}^{*} .
\end{aligned}
$$

Let $N \equiv\left\{\max (n) \mid(m, n) \in A_{\psi} \cup A_{T}\right\}$, i.e., the maximum vertical mode number included in the truncation. If we assume the sum over $p$ runs from $-N^{\prime}$ to $N^{\prime}$, we can write Eq. (9) as

$$
\frac{d}{d t} E=i \alpha \sum_{m} \sum_{n=-N}^{N} \sum_{p=-N^{\prime}}^{N^{\prime}}(-1)^{p} m \psi_{m n} T_{m, n-p}^{*} .
$$

Now replace $p$ by $s=n-p$ :

$$
\frac{d}{d t} E=i \alpha \sum_{m} \sum_{n=-N}^{N} \sum_{s=-N^{\prime}+n}^{N^{\prime}+n}(-1)^{n-s} m \psi_{m n} T_{m, s}^{*},
$$

and note that the maximum lower bound for $s$ is $-N^{\prime}+N$ when $n=N$, while the minimum upper bound is $N^{\prime}-N$ when $n=-N$. If $N^{\prime}=2 N, s \in[-N, N]$ always (since for $|s|>N$ the mode is not included in the truncation and so is made to vanish), and we can use the symmetries given by Eq. (6) to show that $d E / d t$ vanishes. 7 Hence, we must have $p$ running from $-2 N$ to $2 N$, which from Eq. (8b) implies adding the modes $T_{0,-2 N} \ldots T_{0,2 N}$ to $A_{T}$.

For the internal energy, $\langle T\rangle$, the expansion is

$$
\langle T\rangle=\frac{2 i}{\pi} \sum_{p \text { odd }} \frac{T_{0 p}}{p},
$$

and its time derivative in the dissipationless limit is

$$
\frac{d}{d t}\langle T\rangle=\frac{2 \alpha i}{\pi} \sum_{m, n} \sum_{p \text { odd }} m \psi_{m n} T_{m, n-p}^{*} .
$$

Comparing Eq. (13) with Eq. (9), we see that Eq. (13) vanishes under the same condition as the total energy E.

\section{Properties of the Truncations}

To show that the truncated systems obtained in Section ППВ have bounded solutions for all times $t>0$, we consider the quantity $Q$ : 


$$
Q \equiv K+2 \sum_{m, n>0}\left|T_{m n}\right|^{2}+\sum_{n>0}\left(T_{0 n}^{i}-\frac{2}{n}\right)^{2}
$$

where $K$ is the kinetic energy defined previously. The quantity $Q$ is non-negative and it includes all the modes in the truncation such that if any of them diverges, then $Q$ diverges. Thus, if $Q$ is bounded from above then the truncated system has no unbounded solutions. Taking the time derivative of $Q$, with the viscosity and thermal conductivity nonzero, we can write

$$
\frac{d}{d t} Q \leq-\min \{2 \nu, \kappa\} Q+4 \kappa N_{0}
$$

with $N_{0}$ being the number of $T_{0|n|}$ modes included in the truncation. For $Q>4 \kappa N_{0} / \min \{2 \nu, \kappa\}$, we have $d Q / d t<0$, and so $Q$ is bounded. 10

We define the horizontally averaged vertical thermal flux as $q(y) \equiv q_{\mathrm{cv}}(y)+q_{\mathrm{cd}}(y)$, where $q_{\mathrm{cv}}(y)=\overline{v_{y} T}$ is the convective thermal flux and $q_{\mathrm{cd}}(y)=\kappa\left(1-\overline{\partial_{y} T}\right)$ is the conductive thermal flux (the overbar denotes an average over $x$ ). For energy-conserving truncations one can write the expansion for $q$ ast 10

$$
q(y)=\langle q\rangle-2 \sum_{m>0} \frac{\cos m y}{m} \frac{d}{d t} T_{0 n}^{i} .
$$

In a steady-state situation this reduces to the expected result $q=\langle q\rangle$, independent of $y$, showing that the energy cannot "pile up" in steady convection. For a general truncation (for example, the truncations in Refs. 1, 8,99), one cannot write $q$ in the form given by Eq. (16) and the thermal flux has an unphysical $y$ dependence in a steady-state. General truncations can also have unbounded solutions as is the case in Ref. 1 for large enough Rayleigh number.

\section{LOW-ORDER TRUNCATIONS}

A popular truncation of the Boussinesq equations (in the spirit of the Lorenz model 1 ) is the 6-ODE model given by Howard and Krishnamurtit and used by other authors.12 It includes the 6 independent modes $\psi_{01}^{i}, \psi_{11}^{r}, \psi_{12}^{i}, T_{11}^{i}, T_{12}^{r}$, and $T_{02}^{i}$. The Howard and

Krishnamurti truncation is the simplest one that allows for a nonzero shear flow (the $\psi_{01}^{i}$ 
mode). It has a vanishing $\langle T\rangle$. However, it is not energy-conserving: it lacks the $T_{04}^{i}$ mode. We will add this $T_{04}^{i}$ mode to the 6 -ODE model to obtain what we will call the 7-ODE model. Figure 1 displays explicitly the energy-conserving property of the 7-ODE model in the dissipationless limit. Note that the nonconservation of energy for the 6-ODE model is not a small effect: Figure 1 shows that the energy is not conserved by a factor of 1.8 to as much as 2.8 .

Figure 2 shows the time-averaged quantity $\mathrm{Nu} \times R / R_{c}$ evaluated at the upper boundary plotted as a function of the Rayleigh number scaled by $R_{c}$, the critical Rayleigh number where the fluid at rest becomes linearly unstable $\left(R_{c}=\left(1+\alpha^{2}\right)^{3} / \alpha^{2} \simeq 10.088\right.$ for $\left.\alpha=1.2\right)$. The Nusselt number $\mathrm{Nu}$ is the ratio of total heat transferred to the heat conducted when the fluid is at rest, so that $\mathrm{Nu} \times R / R_{c}$ is a dimensionless measure of the thermal flux. 3 The thermal flux in the 6-ODE model (solid line) is seen to grow rapidly after $R / R_{c} \simeq 11$, whereas the same quantity for the 7-ODE model has a slower growth (dashed line). The dotted line has a slope of 5.05, corresponding to the experimental result of Ref. 3 (the intercept is arbitrary) for $\sigma=7$ (the numerical results are also for $\sigma=7$ ). The agreement between the experimental results and that of the 7-ODE model is excellent. However, since the experiment was done with no-slip boundary conditions, caution should be taken in concluding the accuracy of the model (the value of $R_{c}$ used to scale the experimental curve is the no-slip one). The onset of shear flow (at $R / R_{c} \simeq 8.1$ ) is associated with a decrease in thermal flux for the 6-ODE model but an increase of that quantity for the 7-ODE model. For smaller values of $\sigma$, the two models behave in a more similar fashion, as seen in Figure 3 where the same quantities are plotted for $\sigma=1$.

Figure 4 shows the thermal flux $q$ for the 6-ODE model evaluated at different values of $y$ as a function of time $\left(R / R_{c}=3.5, \sigma=1, \alpha=1.2\right)$. The thermal flux after the system settles in a steady-state is seen to depend on $y$, which is unphysical since this would lead to energy pile up (see Section [IIव). However, as shown in Figure 5 the thermal flux for the energy-conserving 7-ODE model is independent of $y$ when the system reaches a steady-state. 


\section{CONCLUSIONS}

In this work we have developed a general method for generating energy-conserving Galerkin approximations of the PDE's that describe Rayleigh-Bénard convection, using a generalization of the method of Treve and Manley. The truncations allow for shear flow (zonal flows independent of the horizontal coordinate, $x$ ) and variable phase of the rolls (breaking point symmetry with respect to the center of the rolls). These flows also have applications in tokamak plasmas, where it is thought that a shear flow in the edge layer is responsible for the so-called H-mode, 13 in which confinement is increased by a factor of two over the normal, or L-mode phase. Convection cells form as a result of the nonlinear development of the Rayleigh-Taylor instability in regions of unfavorable magnetic curvature. Such convection cell turbulence is widely observed in the edge of tokamak plasmas 14 These vortices can lead to the generation of a shear flow in a manner analogous to the Rayleigh-

Bénard case 1516 and it is believed that this flow creates a barrier to particle transport, thereby improving confinement. The new truncations could help to provide a foundation for turbulence models of L-H transitions such as in Ref. 17 .

There are essentially three arguments for using energy-conserving approximations: First, the cascade of energy through the inertial range to the dissipation scale is modeled without extraneous terms in the energy equations. Thus, energy-conserving truncations are a good technique to reduce unphysical numerical dissipation or sources and possible instabilities. This makes the truncations more closely related to the full equations, since in the full PDE's the dissipation comes entirely from the linear terms and the nonlinear parts of the equations conserve energy. Note, however, that for the example given (the 7-ODE model) there are too few modes to speak of an energy cascade through an inertial range. The second property is the correct description of the thermal flux in the steady-state limit, even with dissipation. This means that the fact that a truncation does not conserve energy in the ideal limit significantly affects the way the energy flows in the dissipative regime, and so the energy-conserving truncations are relevant to the dissipative case. Finally, the boundedness 
of solutions is a strong point since physically one does not expect divergent behavior and hence the energy-conserving truncations are more reliable.

In the dissipationless limit, we have demonstrated that energy conservation is violated drastically for a typical truncation (the 6-ODE Howard and Krishnamurti model 1 ), whereas it is conserved to machine precision for the energy-conserving truncation (7-ODE model). In the presence of dissipation, the numerical results for the comparison of the two models for Prandtl number $\sigma=7$ clearly show that the energy-conserving truncation is much closer to experimental results. If the system reaches a steady-state, the 7-ODE model properly models the thermal flux, whereas the 6-ODE model exhibits an unphysical dependence on the vertical coordinate. Thus we believe that energy-conserving truncations represent the

full system more accurately. Note, however, that the 7-ODE model is presented here as an illustration of the energy-conserving truncation technique, as severely truncated systems may yield numerical results far removed from the behavior of the full system. in Ref. 1 the authors concluded that there are not enough modes in the 6-ODE truncation to adequately model the bifurcations observed in a full simulation of the flow. Note also that Ref. 19 gives a different 7-ODE extension of the 6-ODE model that conserves total vorticity in the dissipationless limit. That model could be made energy-conserving by adding to it a $T_{06}^{i}$ mode.

\section{ACKNOWLEDGMENTS}

The authors would like to thank Philip J. Morrison and Neil J. Balmforth for useful discussions. J.-L.T. acknowledges support from the Natural Sciences and Engineering Research Council of Canada. 


\section{REFERENCES}

${ }^{1}$ L. N. Howard and R. K. Krishnamurti, "Large-scale flow in turbulent convection: a mathematical model," J. Fluid Mech. 170, 385 (1986).

${ }^{2}$ S. Chandrasekhar, Hydrodynamic and Hydromagnetic Stability (Dover, New York, 1981).

${ }^{3}$ R. Krishnamurti and L. N. Howard, "Large-scale flow generation in turbulent convection," Proc. Natl. Acad. Sci. USA 78, 1981 (1981).

${ }^{4}$ J. Prat, J. M. Massaguer, and I. Mercader, "Large-scale flows and resonances in 2-D thermal convection," Phys. Fluids 7, 121 (1995).

${ }^{5}$ S. A. Orszag, "Numerical simulations of incompressible flows within simple boundaries. I. Galerkin (spectral) representations," Studies in Appl. Math. L, 293 (1971).

${ }^{6}$ P. J. Morrison and R. D. Hazeltine, "Hamiltonian formulation of reduced magnetohydrodynamics," Phys. Fluids 27, 886 (1984).

${ }^{7}$ Y. M. Treve and O. P. Manley, "Energy conserving Galerkin approximations for 2-D hydrodynamic and MHD Bénard convection," Physica D 4, 319 (1982).

${ }^{8}$ J. H. Curry, "A generalized Lorenz system," Commun. Math. Phys. 60, 193 (1978).

${ }^{9}$ J. H. Curry, J. R. Herring, J. Loncaric, and S. A. Orszag, "Order and disorder in twoand three-dimensional Bénard convection," J. Fluid Mech. 147, 1 (1984).

${ }^{10}$ J.-L. Thiffeault, Master's thesis, University of Texas at Austin, 1995, Institute for Fusion Studies Report \#715.

${ }^{11}$ E. N. Lorenz, "Deterministic nonperiodic flow," J. Atmos. Sci. 20, 130 (1963).

${ }^{12}$ A. M. Rucklidge and P. C. Matthews, "Analysis of the shearing instability in nonlinear convection and magnetoconvection", 1995, submitted to Nonlinearity.

${ }^{13}$ F. Wagner et al., "Regime of improved confinement and high beta in neutral-beam-heated 
divertor discharges of the ASDEX tokamak," Phys. Rev. Lett. 49, 1408 (1982).

${ }^{14}$ A. J. Wooton, B. A. Carreras, H. Matsumoto, K. McGuire, W. A. Peebles, C. P. Ritz, P. W. Terry, and S. J. Zweben, "Fluctuations and anomalous transport in tokamaks," Phys. Fluids B 2, 2879 (1990).

${ }^{15}$ J. F. Drake, J. M. Finn, P. N. Guzdar, V. Shapiro, V. Shevchenko, F. Waelbroeck, A. B. Hassam, C. S. Liu, and R. Sagdeev, "Peeling of convection cells and the generation of sheared flows," Phys. Fluids B 4, 488 (1992).

${ }^{16}$ J. M. Finn, J. F. Drake, and P. N. Guzdar, "Instability of fluid vortices and generation of sheared flows," Phys. Fluids B 4, 2758 (1992).

${ }^{17}$ H. Sugama and C. W. Horton, "L-H confinement mode dynamics in three-dimensional state space," Plasma Phys. Control. Fusion 37, 345 (1995).

${ }^{18}$ C. Foias, O. P. Manley, R. Temam, and Y. M. Treve, "Asymptotic analysis of the NavierStokes equations," Physica D 9, 157 (1983).

${ }^{19}$ K. B. Hermiz, P. N. Guzdar, and J. M. Finn, "Improved low-order model for shear flow driven by Rayleigh-Bénard convection," Phys. Rev. E 51, 325 (1995). 


\section{FIGURES}

FIG. 1. Total energy $E$ in the dissipationless limit $(\nu=\kappa=0)$ for the 6-ODE (solid line) and 7-ODE (dashed line) models with $\alpha=1.2$ and initial conditions (a) $\psi_{11}^{r}=\psi_{01}^{i}=\psi_{12}^{i}=T_{11}^{i}=1$; all other modes begin at $10^{-5}$, (b) $\psi_{11}^{r}=T_{11}^{i}=1$; all other modes begin at $10^{-8}$.

FIG. 2. Time-averaged $\mathrm{Nu} \times R / R_{c}$ evaluated at $y=\pi$ as a function of $R / R_{c}$ for the 6 -ODE (solid line, triangles) and 7-ODE (dashed line, black dots) models. The dotted line has a slope of 5.05, corresponding to the experimental results for $\sigma=7$ in Ref. 3 .

FIG. 3. Time-averaged $\mathrm{Nu} \times R / R_{c}$ evaluated at $y=\pi$ as a function of $R / R_{c}$ for the 6-ODE (solid line, triangles) and 7-ODE (dashed line, black dots) models.

FIG. 4. Thermal flux $q$ for the 6-ODE model evaluated at different values of $y$ as a function of time. The final, steady-state thermal flux is seen to depend on $y$.

FIG. 5. Thermal flux $q$ for the 7-ODE model evaluated at the same values of $y$ as in Figure 1 as a function of time. The final, steady-state thermal flux is independent of $y$ as expected physically. 

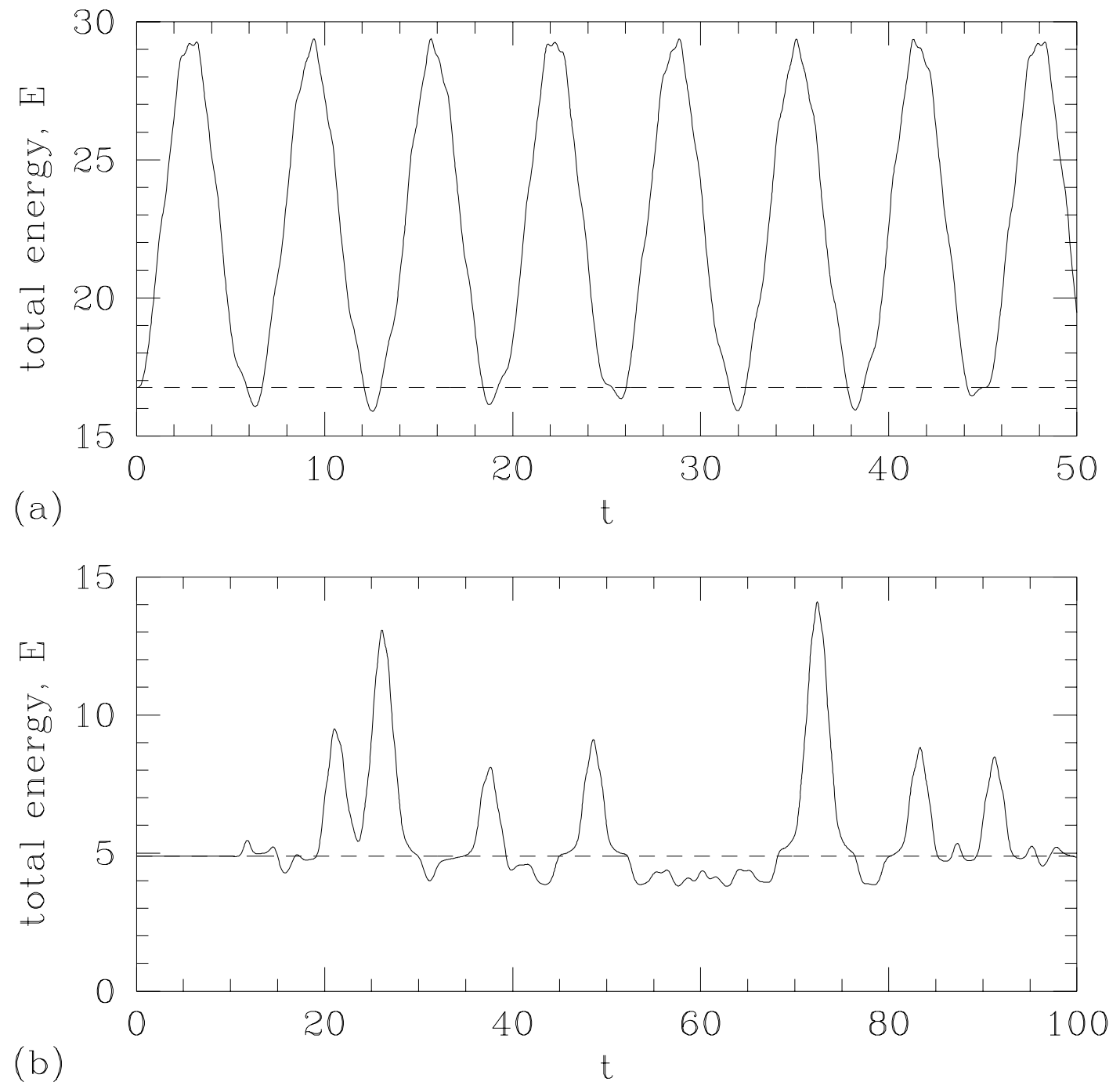

Thiffeault and Horton, Fig. 1 


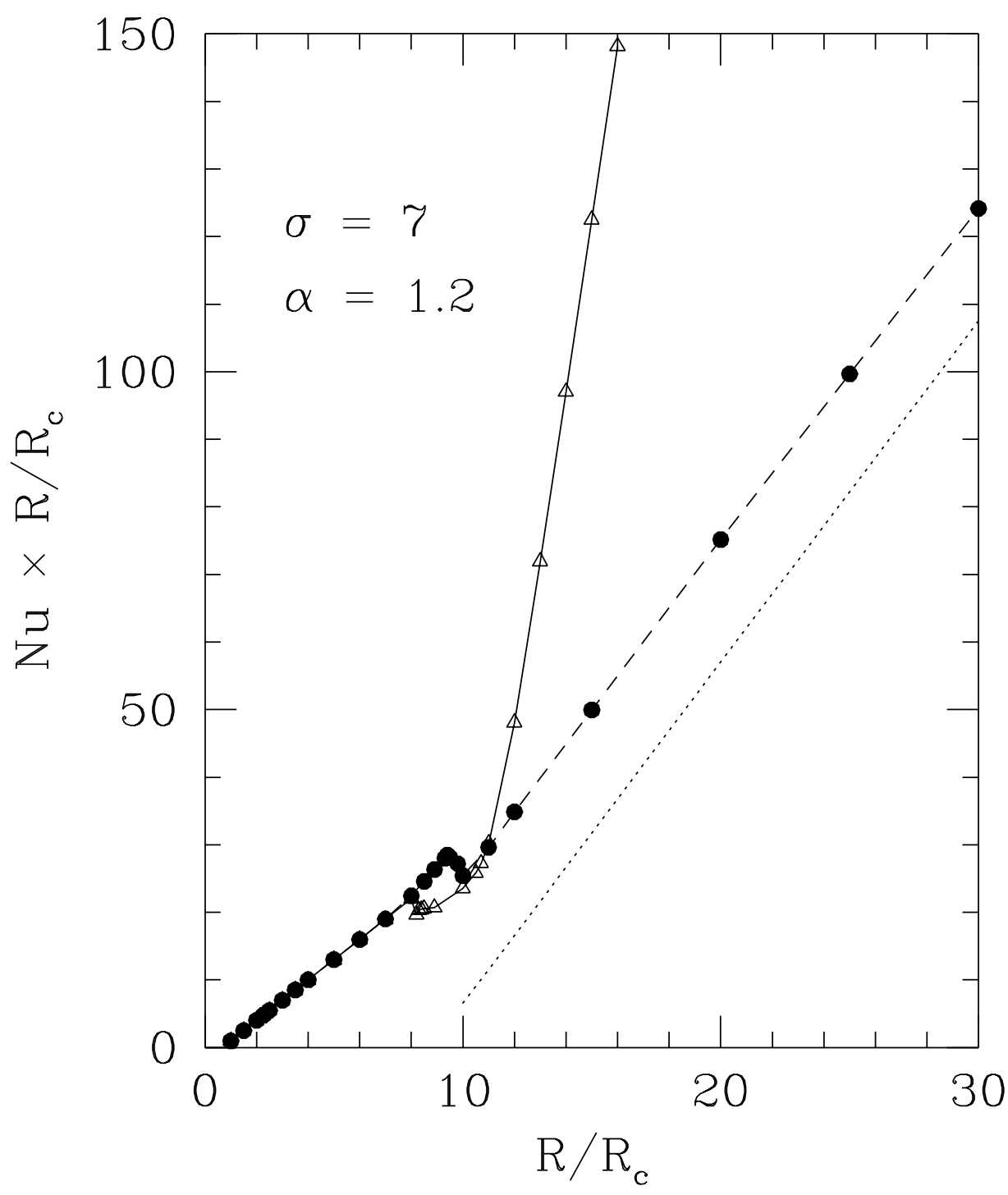

Thiffeault and Horton, Fig. 2 


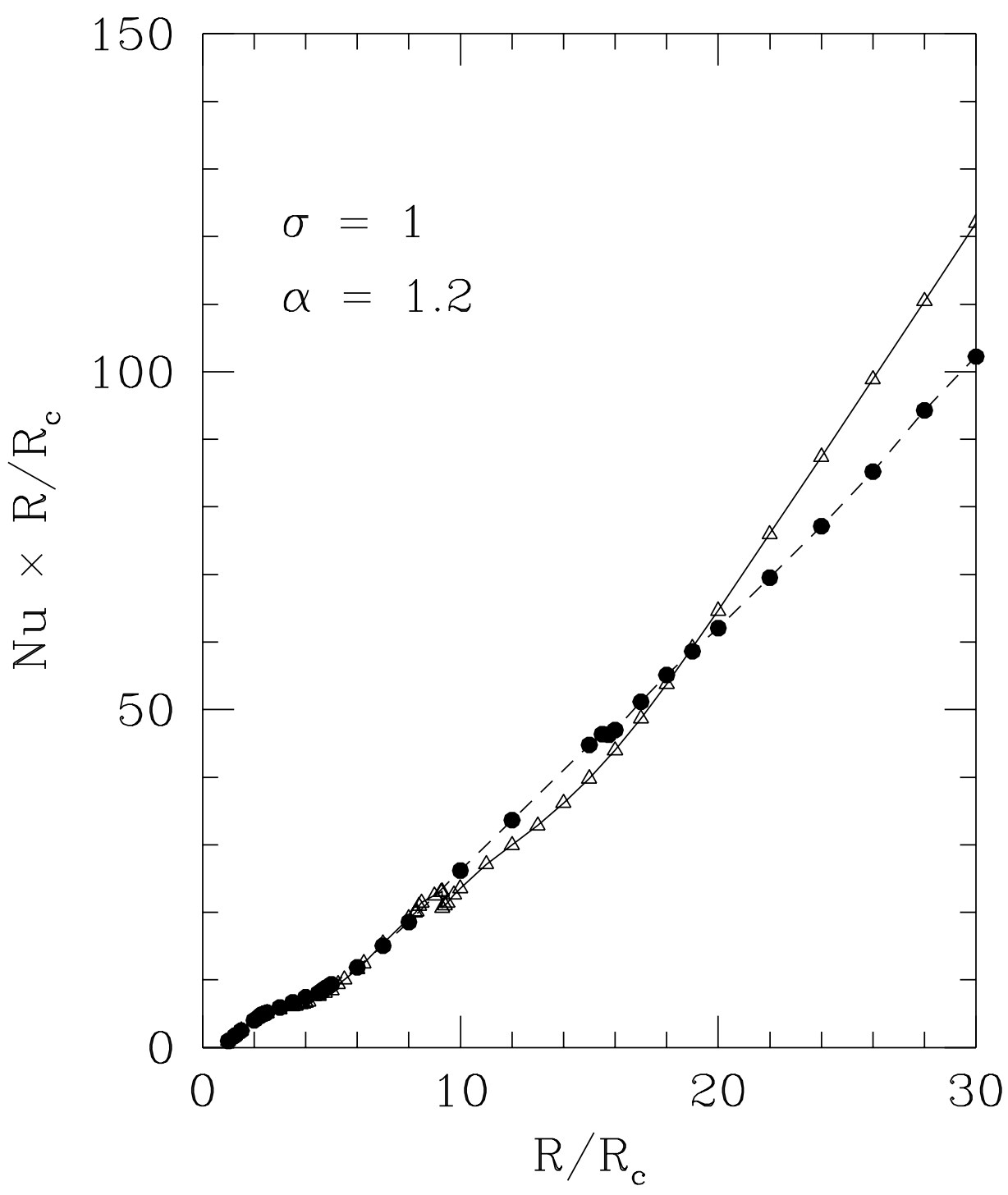

Thiffeault and Horton, Fig. 3 


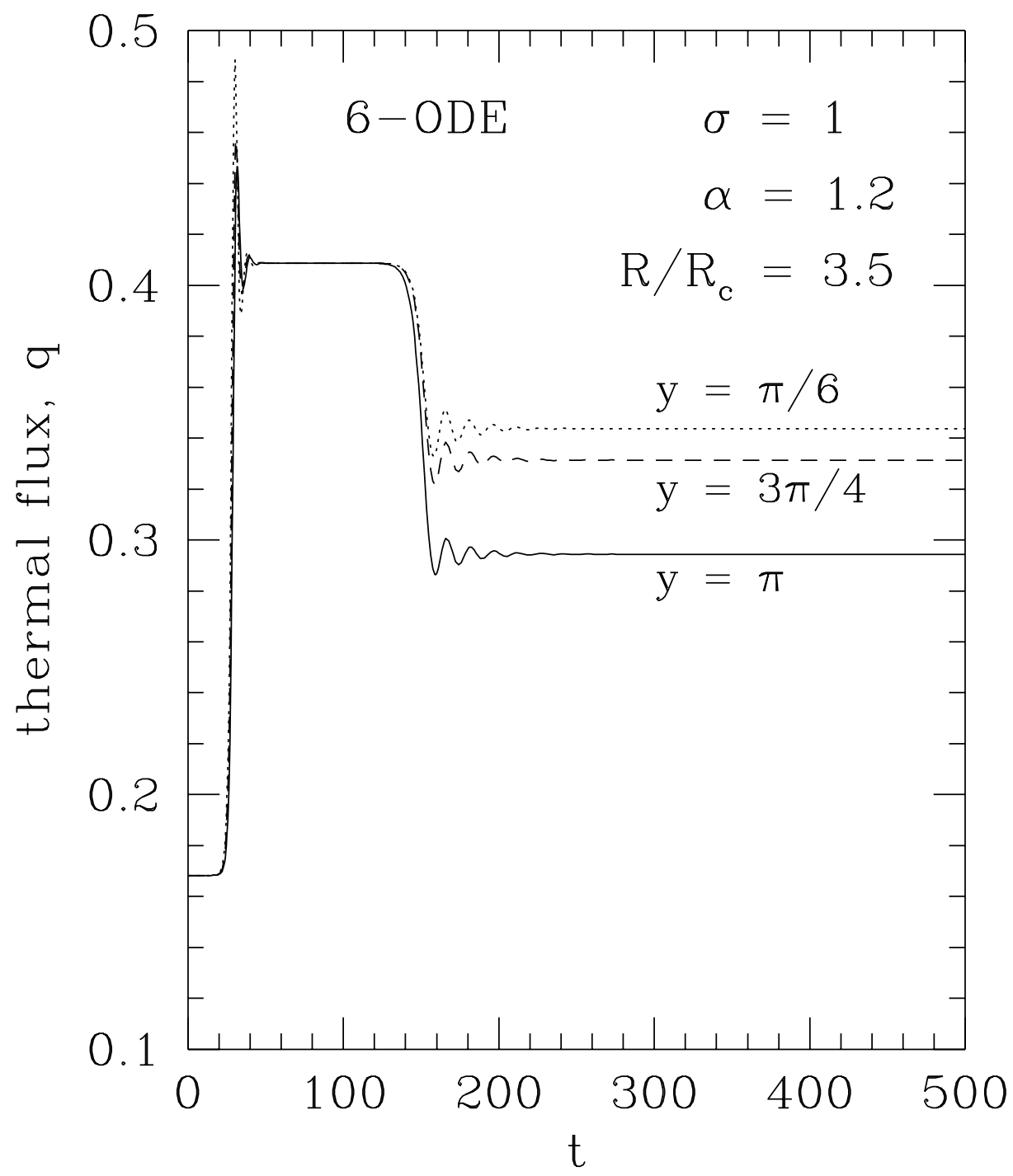

Thiffeault and Horton, Fig. 4 


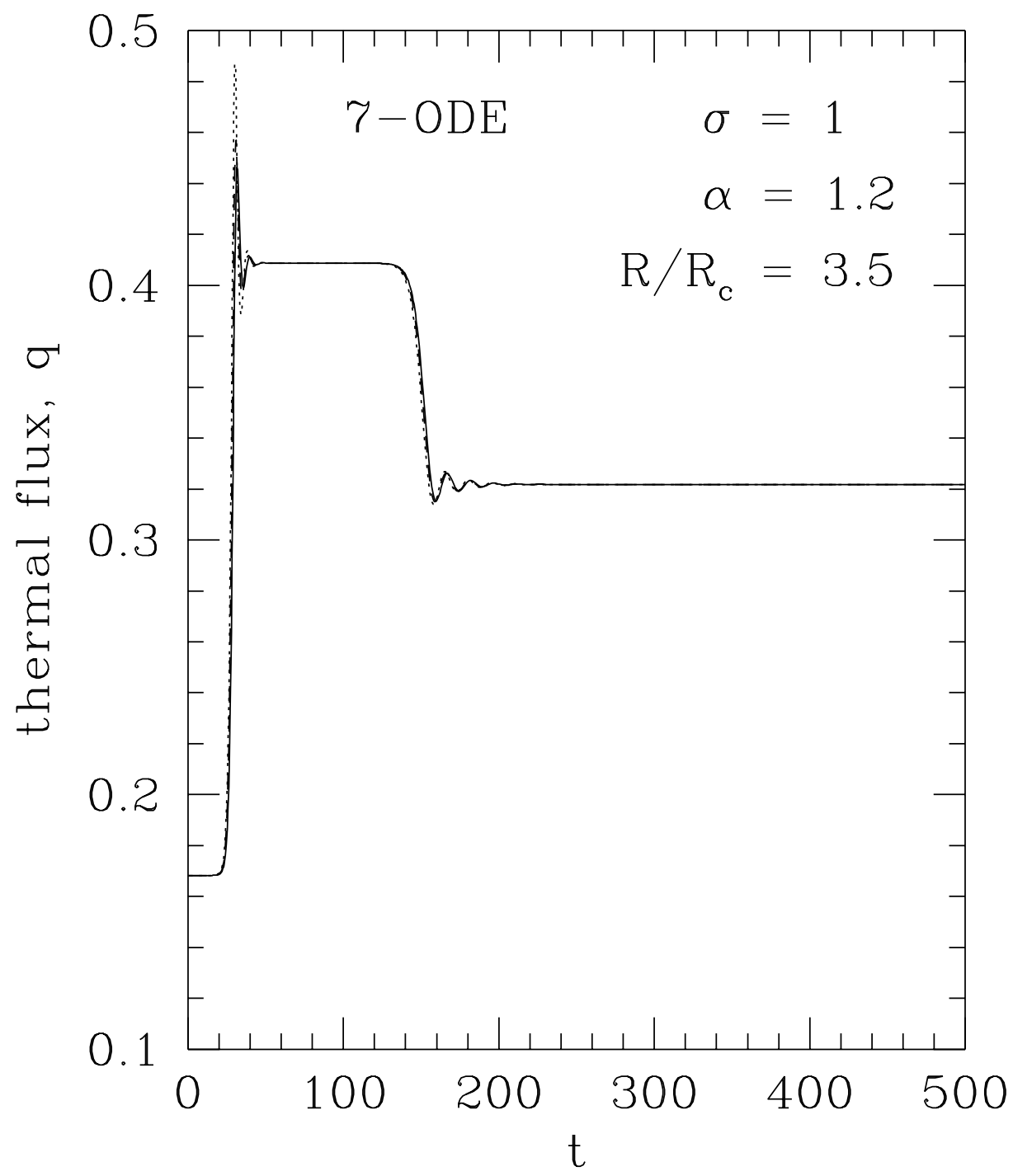

Thiffeault and Horton, Fig. 5 